\title{
Patients' expectations of deep brain stimulation, and subjective perceived outcome related to clinical measures in Parkinson's disease: a mixed-method approach
}

\author{
Franziska Maier, ${ }_{1}^{1}$ Catharine J Lewis, ${ }^{1}$ Nina Horstkoetter, ${ }^{2}$ Carsten Eggers, ${ }^{1}$ \\ Elke Kalbe, ${ }^{1,3}$ Mohammad Maarouf, ${ }^{4}$ Jens Kuhn, ${ }^{5}$ Mateusz Zurowski, ${ }^{6}$ Elena Moro, ${ }^{7}$ \\ Christiane Woopen, ${ }^{2}$ Lars Timmermann ${ }^{1}$
}

- Additional material is published online only. To view please visit the journal online (http://dx.doi.org/10.1136/ jnnp-2012-303670)

${ }^{1}$ Department of Neurology, University of Cologne, Cologne, Germany

${ }^{2}$ Research Unit Ethics, Institute for the History of Medicine and Medical Ethics, University of Cologne, Cologne, Germany ${ }^{3}$ Institute for Gerontology, University of Vechta, Vechta, Germany

${ }^{4}$ Department of Stereotaxy and Functional Neurosurgery, University of Cologne, Cologne, Germany

${ }^{5}$ Department of Psychiatry and Psychotherapy, University of Cologne, Cologne, Germany ${ }^{6}$ Department of Psychiatry, Department of Medicine, University of Toronto, University Health Network Toronto, Ontario, Canada Movement Disorders Unit, Department of Psychiatry and Neurology, University Hospital Center (CHU) of Grenoble, Grenoble, France

\section{Correspondence to}

Franziska Maier, Department of Neurology, University Hospital Cologne, Kerpener Str. 62, Cologne D-50937, Germany; franziska.maier@uk-koeln.de

FM and CJL contributed equally.

Received 13 July 2012

Revised 2 April 2013

Accepted 1 May 2013

Published Online First

28 May 2013

To cite: Maier $F$, Lewis CJ Horstkoetter $\mathrm{N}$, et al. J Neurol Neurosurg Psychiatry 2013;84:1273-1281.

\section{ABSTRACT}

Objective To study patients' expectations of subthalamic deep brain stimulation (STN-DBS) and their subjective perceived outcome, by using qualitative and quantitative methods in Parkinson's disease (PD).

Methods PD patients were prospectively examined before and 3 months after surgery. Semistructured interviews regarding preoperative expectations and postsurgical subjective perceived outcome were conducted. These were analysed using content analysis. For statistical analyses, patients were classified according to their subjective perceived outcome, resulting in three different subjective outcome groups (negative, mixed, positive outcome). The groups were used for multiple comparisons between and within each group regarding motor impairment, quality of life (QoL), neuropsychiatric status and cognitive functioning, using standard instruments. A logistic regression analysis was conducted to find predictors of subjective negative outcome. Receiver operating characteristic curves were used to analyse cut-off scores for predictive tests.

Results Of the 30 PD patients participating, 8 had a subjective negative outcome, 8 a mixed and 14 a positive outcome. All groups significantly improved in motor functioning. Patients with subjective negative outcome were characterised by preoperative unrealistic expectations, no postsurgical improvement in QoL, and significantly higher presurgical and postsurgical apathy and depression scores. Higher preoperative apathy and depression scores were significant predictors of negative subjective outcome. Cut-off scores for apathy and depression were identified.

Conclusions The mixed-method approach proved useful in examining a patient's subjective perception of STN-DBS outcome. Our results show that significant motor improvement does not necessarily lead to a positive subjective outcome. Moreover, PD patients should be screened carefully before surgery regarding apathy and depression. (DRKS-ID: DRKS00003221).

\section{INTRODUCTION}

Parkinson's disease (PD) is a neurodegenerative disorder characterised by a variety of typical motor and non-motor impairments. While bilateral subthalamic deep brain stimulation (STN-DBS) significantly improves the motor difficulties, ${ }^{1}$ there is an ongoing discussion on how STN-DBS influences behavioural and cognitive symptoms. ${ }^{2}{ }^{3} \mathrm{PD}$-specific quality of life (QoL), measured with the Parkinson's Disease Questionnaire-39 (PDQ-39), ${ }^{4}$ is an often used outcome parameter for STN-DBS. By using this scale, a significant improvement of QoL under STN-DBS has been demonstrated. ${ }^{1} 5$ This finding stands in contrast with reports on patients who perceive their outcome as disappointing, even though they had experienced significant motor benefits and improvement in QoL. ${ }^{6}$ More in-depth examinations of QoL following STN-DBS have revealed a predominant improvement of physical domains, while mental subscales remain stable or even worsen. ${ }^{7}$ The clinical need of identifying patients who are at higher risk of perceiving their postoperative outcome as unsatisfactory has attracted more attention lately. ${ }^{69} 9 \mathrm{By}$ focussing on the patient, individual realistic and unrealistic expectations can be considered. To gain information about this, the combination of qualitative patient interviews with standard measures for a better understanding of subjective outcome might be a successful approach. ${ }^{10}{ }^{11}$ Concerning research on STN-DBS in PD patients, only a few studies used well-appreciated standard instruments (eg, the PDQ-39), as well as supplementary semistructured or open in-depth interviews to explore patient's subjective perception of DBS therapy. ${ }^{6} 12$

Predictors or influencing factors of psychosocial outcome and improved QoL after STN-DBS have been tried to be identified by using standard scales $^{13-16}$ or modified standard scales. ${ }^{9}$ Postsurgical improved QoL has been linked to preoperative cumulative daily off time, ${ }^{13}$ high 1-dopa response, ${ }^{14}$ changes in depression and anxiety, ${ }^{13}$ and better cognitive functioning. ${ }^{16}$ Furthermore, neuropsychiatric symptoms, such as depression and apathy, have a major impact on QoL in PD. ${ }^{17} 18$

In this study, we used a mixed-method approach of qualitative and quantitative methods to examine the patient's expectations of STN-DBS and perceived outcome at 3 months after surgery. As primary goal, we intended to analyse the number of patients who perceived their subjective outcome as negative, mixed, or positive. Secondarily, we sought to characterise differences between these patients by using standard clinical measures. Finally, we aimed to examine the predictive value 
of preoperative scores for patients with subjective negative outcome.

\section{METHODS}

\section{Patients}

We prospectively examined patients with idiopathic PD, recruited from the Department of Neurology, University Hospital Cologne, before surgery (baseline) and 3 months after STN-DBS (3 mFU). All patients met the criteria for STN-DBS treatment according to the guidelines of the German Neurological Society. ${ }^{19}$ Included were patients between 40 and 75 years of age who had medication refractory motor symptoms despite an unequivocal response to l-dopa in the off-state.

Patients with dementia (DemTect-score $<9^{20}$ and MMSE-score $<25^{21}$ ), severe psychiatric or additional neurological disorders were excluded. The study was approved by the ethics commission of the University Hospital of Cologne, and all patients gave written informed consent. Furthermore, the study was registered at the German Clinical Trials Register (DRKS-ID: DRKS00003221).

\section{Procedure}

As part of the preoperative DBS screening routine in Cologne, all patients were examined by a movement disorder neurologist, a neurosurgeon, a psychiatrist and a neuropsychologist. These experts mutually approved DBS surgery for all patients. For the purpose of this study, patients were additionally interviewed, examined and cognitively tested 6 weeks to 3 days prior to surgery, and 3 months after implantation of the electrodes, by two trained clinical neuropsychologists (F.M. \& C.L.).

\section{DBS surgery}

All patients underwent STN-DBS surgery at the department for Stereotaxy and Functional Neurosurgery, University Hospital Cologne. Preoperative stereotactic CTs and 1.5T MRIs determined the coordinates of the target structure. Electrodes (standard model 3389; Medtronic, Minneapolis, Minnesota, USA) were implanted bilaterally in the STN under local anaesthesia. Microelectrode recordings and macroelectrode test stimulation determined the optimal implantation area in all patients. To monitor the final location of the electrodes, intraoperative and postoperative stereotactic $\mathrm{X}$-rays were projected on the preoperative stereotactic MRI scans. The electrodes were connected to a pulse generator (Kinetra or Activa RC/PC; Medtronic, Minneapolis, Minnesota, USA) and implanted in the subclavicular area under general anaesthesia. Postoperatively, patients were hospitalised for up to 10 days, according to the standards of care of our centre. STN-stimulation was turned on 3-5 days after surgery on very low amplitudes $(0.3-0.5 \mathrm{~V}, 60 \mu \mathrm{s}, 130 \mathrm{~Hz})$, allowing for immediate microlesion effects to wear off. The main microlesion effect decreased around day 6-10 after surgery. Stimulation amplitude was slowly increased accompanied by a stepwise reduction of antiparkinsonian medication.

\section{Semistructured interviews}

In addition to the quantitative data collection, we intended to gain knowledge about the patient's subjective perspective on their disease and DBS. We developed an interview guideline including various different domains that may have an impact on a patient's life with PD and DBS. Preoperatively, the interview focus was set on the following topics: subjective expectations from DBS, presurgical motor, emotional, social, behavioural and cognitive functioning, activities of daily living and QoL. At $3 \mathrm{mFU}$, the interview contained questions about the impact of
DBS on the same domains. The interviews were pretested with five patients to prove understanding and mental resilience. All 60 interviews ( 30 baseline and $303 \mathrm{mFU}$ ) were recorded, transcribed and categorised by two independent trained coders $(\mathrm{N}$. H. \& C.L.). Categorisation was conducted according to Mayring's theory-based content analysis, ${ }^{22}{ }^{23}$ which is a widely used method in health research. For the purpose of this study, categories were analysed with respect to the patient's expectations from DBS before surgery, and the patient's perception of their personal outcome at $3 \mathrm{mFU}$. At baseline, all expectations that were stated by each patient throughout the interview were collected. At $3 \mathrm{mFU}$, all statements regarding the impact of DBS on the patient's life, such as improved or worsened symptoms or unexpected changes, were collected for each patient. Thereafter, the number of positive and negative statements related to DBS at $3 \mathrm{mFU}$ was separately summed up, and a quotient was calculated by dividing the number of positive statements by the number of negative statements for each patient (eg, 12 positive divided by 3 negative statements is 4). Neutral responses, such as 'no change' answers, were not considered. Finally, patients were grouped according to their subjective outcome quotient: group 1 included patients with a quotient of $\leq 0.5$, representing patients who had at least twice as many negative statements than positive statements. Therefore, the first group contained patients who perceived their subjective outcome as mainly negative (group-neg). The second group included patients with a quotient between $>0.5$ and $<2.0$, representing patients who considered their outcome as mixed positive and negative (group-mix). The third group contained patients with a quotient of $\geq 2$, representing patients who had at least twice as many positive statements than negative statements and, thus, perceived their subjective outcome as mainly positive (group-pos).

\section{Clinical rating scales}

Motor performance was evaluated using the Unified Parkinson's Disease Rating Scale part III (UPDRS-III) ${ }^{24}$ while on and off 1-dopa. Off-state was defined as motor impairment following at least $12 \mathrm{~h}$ absence of antiparkinsonian medication, while on-state was the patient's best motor response to first 1-dopa dose (at least $200 \mathrm{mg}$ or 1.5 times the individual morning dose) after the off-state. Long-acting dopamine agonists were stopped $72 \mathrm{~h}$ prior to off-examination. Also, the 1-dopa equivalent daily dose (LEDD) ${ }^{25}$ was determined. At $3 \mathrm{mFU}$, patients were tested on the UPDRS-III with stimulation on and medication off.

Regarding neuropsychological testing, all patients were examined on the digit span forwards and backwards of the Wechsler memory scale, ${ }^{26}$ phonemic verbal fluency task (letter B, M, S), ${ }^{27}$ and the brief test of attention (BTA). ${ }^{28}$

To determine patient's QoL, the Parkinson's disease questionnaire-39 (PDQ-39) ${ }^{4}$ was given to all patients at baseline (PDQ-base) and at $3 \mathrm{mFU}$ (PDQ-3 $\mathrm{mFU}$ ).

With respect to mood examination, the following tests were conducted: apathy evaluation scale (AES, range: 18-72), ${ }^{29}$ Beck depression inventory-2 (BDI-2, range: $0-63),{ }^{30}$ self-report manic inventory (SRMI, range: $0-48),{ }^{31}$ and the state subscale of the State trait anxiety inventory (STAI-state, range: 20-80). ${ }^{32}$ For all four scales, higher scores represent more severe symptoms.

\section{Monitoring}

All data was transferred into a SPSS database and independently controlled by the PI. To ensure data quality, the Cologne Centre for Clinical Studies (ZKS Cologne), an independent clinical 
research organisation, monitored $20 \%$ of the data. The data quality was rated as very high by the ZKS Cologne.

\section{Statistical analysis}

All statistical analyses were conducted using IBM SPSS V.20.0 (SPSS, Chicago, Illinois, USA). Level of significance was set at 0.05 . To ensure standard treatment outcome, the whole sample was compared regarding baseline and $3 \mathrm{mFU}$ UPDRS-III-scores and PDQ-scores using paired samples $t$ tests (assumptions for parametric distribution were fulfilled).

All patients were classified according to their individual outcome quotient into three subjective outcome groups (group-neg/-mix/-pos).

\section{Group differences}

First, the three groups were compared concerning presurgical expectations, and postoperative positive and negative statements using the $\chi^{2}$ test or the Fisher's exact test when one value was not available. Thereafter, for clinical tests, differences between the subjective outcome groups were analysed separately at baseline and at $3 \mathrm{mFU}$ with one-way ANOVAs for parametric variables and with the Kruskall-Wallis $\mathrm{H}$ test for nonparametric variables. Significant $\mathrm{p}$ values were $\alpha$-corrected by dividing 0.05 by the number of tests for each analysis. Posthoc Bonferroni test was used to further specify significant group differences. To compare baseline and $3 \mathrm{mFU}$ data within each group, paired samples t tests were computed. Neurological data, neuropsychological measurements, QoL, and mood scales were analysed separately.

\section{Predictors of subjective negative outcome}

A binary logistic regression model was used (forward; LR method), to analyse predictors for group-neg. For this analysis, we only included test scores which showed significant group differences between group-neg and group-mix or/and group-pos at baseline. Because we mainly focussed on patients of group-neg, the dichotomised dependent variable comprised group-neg versus a cluster of group-mix and group-pos.

Exploratively, to determine possible clinically relevant cut-off scores for significant predictors, receiver operating characteristic (ROC) curves were conducted and the area under the curve (AUC) was calculated. Also, sensitivity and specificity were examined for each cut-off score.

\section{RESULTS}

\section{Sample}

Thirty patients with PD were enrolled in this study. Mean age of all patients (18 men/12 women) was $61.20 \pm 8.65$ with an average disease duration of $11.97 \pm 6.79$ years. Standard treatment outcome was classified as good, as patient's baseline UPDRS-III off-score $(42.48 \pm 13.07)$ significantly improved under stimulation at $3 \mathrm{mFU}$ (UPDRS-III stimulation on and medication off: $26.76 \pm 13.86 ; \mathrm{p}<0.001$, paired samples $t$ test). Similarly, the PDQ-base improved from $37.01 \pm 16.54$ for all patients to PDQ-3 mFU 26.47 $\pm 14.94 \quad(\mathrm{p}<0.001$, paired samples $t$ test).

According to the subjective outcome quotient, 8 patients (26.67\%) were assigned to group-neg, 8 patients (26.67\%) were classified as group-mix, and 14 patients (46.66\%) were assigned to group-pos (see table 1). Regarding the three subjective outcome groups, no significant differences were found for demographic data and global cognitive state.

\section{Expectations from DBS and subjective outcome reports}

All patients expected an improvement of motor symptoms (see figure 1) while all other expectations varied between the subjective outcome groups. Group-neg patients significantly more often expected 'improvement of mental state' compared with the other two groups $(p=0.028)$. Moreover, although not significant, group-neg more often expected 'more socialising' or 'improvement of partnership', which can usually not be directly improved by STN-DBS (for details see online supplementary). With respect to patient's subjective outcome at $3 \mathrm{mFU}$ (see figures 2 and 3), all patients of group-mix and group-pos experienced 'improved motor symptoms' which was significantly more often compared with group-neg $(\mathrm{p}=0.010)$. Also, all patients of group-pos, and $87.5 \%$ of group-mix named QoL as being improved, which was significantly more often compared with group-neg $(p<0.001)$. Regarding negative statements, group-neg significantly more often reported a worse mental state than the other two groups $(p=0.002)$ (see online supplementary data).

\section{Subjective outcome group differences at baseline and $3 \mathrm{mFU}$}

Results of the outcome group differences are depicted in tables 2 and 3.

Neurological data

Corrected levels of significance were determined for baseline $(p=0.017 ; 0.05$ divided by 3$)$ and $3 \mathrm{mFU}(p=0.025 ; 0.05$

Table 1 Group classification, demographic data, and global cognitive functioning of the three outcome groups

\begin{tabular}{|c|c|c|c|c|c|}
\hline & Group-neg & Group-mix & Group-pos & $F / \chi^{2}$ & $\mathrm{p}$ Value \\
\hline N (\%) & $8(26.67)$ & $8(26.67)$ & $14(46.66)$ & & \\
\hline Number of positive statements & $1.88 \pm 1.25$ & $5.75 \pm 1.67$ & $8.71 \pm 1.86$ & $42.971^{\mathrm{a}}$ & $<0.001$ \\
\hline Number of negative statements & $7.25 \pm 1.91$ & $5.13 \pm 2.23$ & $1.29 \pm 1.49$ & $29.875^{\mathrm{a}}$ & $<0.001$ \\
\hline Subjective outcome quotient & $0.26 \pm 0.14$ & $1.23 \pm 0.37$ & $6.45 \pm 3.48$ & $21.009^{a}$ & $<0.001$ \\
\hline Age (yr) & $60.50 \pm 7.19$ & $58.88 \pm 9.33$ & $62.93 \pm 9.24$ & $0.577^{\mathrm{a}}$ & 0.568 \\
\hline $\operatorname{Sex}(m / f)$ & $5 / 3$ & $3 / 5$ & $10 / 4$ & $2.470^{\mathrm{b}}$ & 0.291 \\
\hline Education (yr) & $11.75 \pm 4.71$ & $11.38 \pm 4.44$ & $11.29 \pm 4.20$ & $0.006^{\mathrm{b}}$ & 0.997 \\
\hline Disease duration (yr) & $11.00 \pm 4.78$ & $14.38 \pm 8.14$ & $11.14 \pm 7.07$ & $0.672^{\mathrm{a}}$ & 0.519 \\
\hline Mini mental state exam & $28.88 \pm 0.64$ & $29.50 \pm 1.07$ & $28.36 \pm 1.98$ & $3.178^{b}$ & 0.204 \\
\hline DemTect & $13.38 \pm 2.92$ & $14.75 \pm 3.65$ & $15.57 \pm 2.41$ & $1.449^{a}$ & 0.252 \\
\hline
\end{tabular}

Values are means $( \pm S D)$ unless stated otherwise. $p$ Values by ${ }^{a}$ ANOVA $(F)$ or ${ }^{b}$ Kruskall-Wallis H-test $\left(\chi^{2}\right)$.

$\mathrm{N}$, number of patients; group-neg, subjective outcome negative; group-mix, subjective outcome mixed; group-pos, subjective outcome positive. 
group 1: subjective outcome negative $\square$ group 2: subjective outcome mixed $\square$ group 3: subjective outcome positive

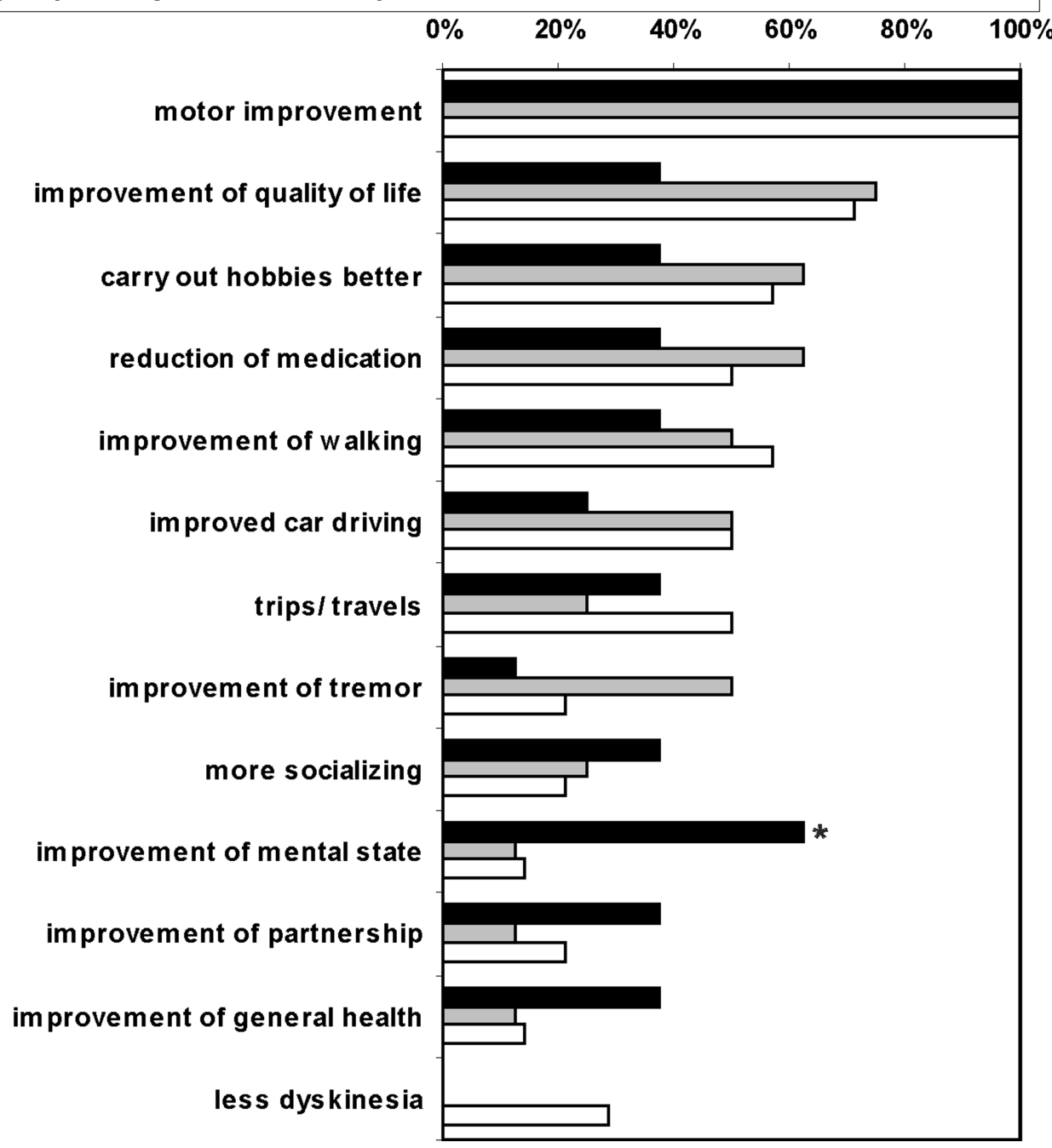

Figure 1 Patient's expectations concerning STN-DBS for the three groups. All patients were asked to name their expectations regarding STN-DBS in a semistructured interview. Expectations were sorted according to their frequency of being stated by all patients and then classified concerning the three subjective outcome groups. Included were only such statements which were named by $>25 \%$ of patients within one of the three groups. * Significant difference between the subjective negative outcome group and the other two groups.

divided by 2). No significant group differences were found for UPDRS-III on-score and off-score, and LEDD at baseline and $3 \mathrm{mFU}$. Within the groups, paired samples t tests revealed significant improvement of UPDRS-III scores (baseline med off vs $3 \mathrm{mFU}$ stim on/med off) and reduced LEDD for each group.

\section{Neuropsychological measures}

Applying corrected levels of significance (baseline and $3 \mathrm{mFU}$ : $\mathrm{p}=0.0125$ ) no group differences were found in any cognitive test results. Within the groups, verbal fluency significantly worsened in group-neg. All other within-group changes were not significant.

\section{Quality of life}

No significant group difference was found for the PDQ-base, although group-neg had the worst QoL. At $3 \mathrm{mFU}$, PDQ-scores were significantly higher in group-neg compared with groupmix and group-pos. Within-group changes revealed that QoL only significantly improved in group-mix and group-pos.
Mood scales

One-way ANOVAs (see table 3) showed significant group differences on the AES and BDI-2 at baseline and $3 \mathrm{mFU}$ applying a corrected level of significance of 0.0125 (0.05 divided by 4 for both time points). Apathy scores were significantly worse in group-neg compared with group-mix and group-pos, verified by posthoc Bonferroni test, at baseline and $3 \mathrm{mFU}$. Depression scores were significantly higher in group-neg compared with group-mix at baseline, and significantly higher compared with group-mix and group-pos at $3 \mathrm{mFU}$. State anxiety of the STAI tended to be higher in group-neg compared with group-mix at baseline, and higher in group-neg compared with group-mix and group-pos at $3 \mathrm{mFU}$. No significant result was found for the SRMI at baseline and $3 \mathrm{mFU}$. Paired samples t tests showed that only in group-pos, depression significantly improved at $3 \mathrm{mFU}$.

Predictors of subjective negative outcome

Baseline group comparisons revealed AES-scores and BDI-2-scores as showing significant differences between group- 


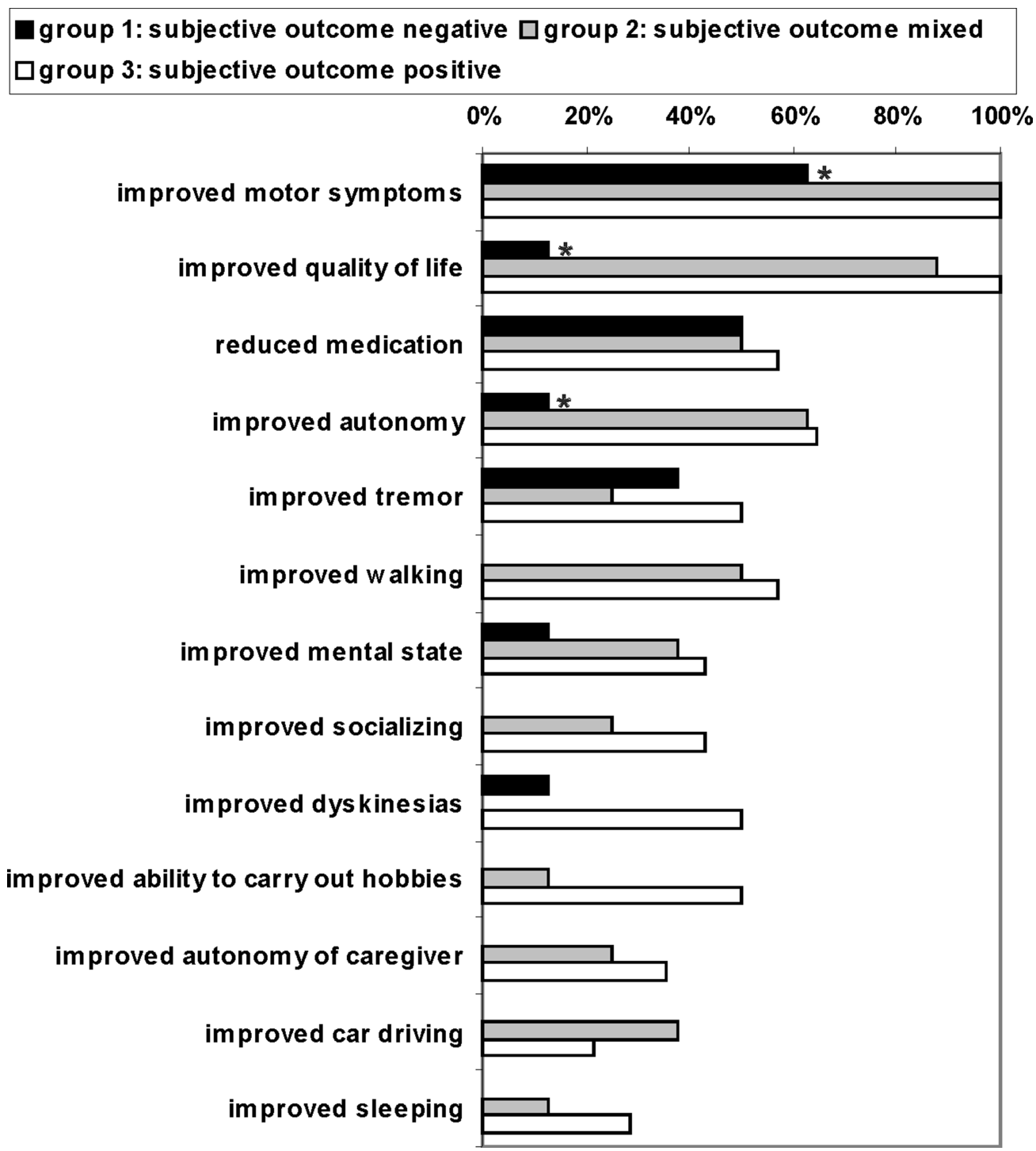

Figure 2 Positive statements at 3 months after STN-DBS for the three groups. All patients were asked to reflect their subjective outcome regarding STN-DBS in a semistructured interview. Positive statements were sorted according to their frequency of being stated by all patients, and were then classified concerning the three subjective outcome groups. Included were only such statements which were named by $>25 \%$ of patients within one of the three groups. *Significant difference between the subjective negative outcome group and the other two groups.

neg $(\mathrm{N}=8)$ and the other groups $(\mathrm{N}=22)$. Therefore, AES and BDI-2 were included in the binary logistic regression analysis. Both tests remained as significant predictors and accounted for $65.8 \%$ of the variance (Nagelkerkes $\mathrm{R}^{2}$ ). This model correctly classified 27 of 30 patients (90\%), applying a criterion value of 0.5 . The sensitivity to find patients with negative subjective outcome (true positive cases) was $87.5 \%$ (7 out of 8 ), while the specificity (true negative cases) was $90.9 \%$ (20 out of 22 ). The AUC was 0.86 for the AES ( $p=0.003,95 \%$ CI 0.71 to 1.00 ) and 0.78 for the BDI- $2(p=0.022,95 \%$ CI 0.55 to 1.00$)$. The ROC curves are shown in figure 4 . The optimal cut-off score (best trade-off between sensitivity and specificity) on the AES was $36 / 37$ (sensitivity $=0.75$, specificity $=0.73$ ), and a score of $16 / 17$ on the BDI-2 (sensitivity $=0.75$, specificity $=0.86$ ). To reduce the false positive rate (1-specificity), a higher cut-off score can be determined. For the AES, a score of 42/43 (sensitivity $=0.63$, specificity $=0.91$ ) and for the BDI-2 a score of $17 / 18$ (sensitivity $=0.63$, specificity $=0.91$ ), reduces the probability of false positives.

\section{DISCUSSION}

This is the first study reporting data on PD patients' expectations of STN-DBS, and on how patients subjectively perceive their outcome at 3 months after surgery. Clearly, all patients expected motor improvement from DBS, and the majority expected improvement in QoL. At $3 \mathrm{mFU}, 14$ patients reported a subjective positive outcome, 8 patients a mixed outcome, and 8 patients a subjective negative outcome. The latter had significantly higher apathy and depression scores at baseline and $3 \mathrm{mFU}$, even though motor scores significantly improved. Also, binary logistic regression analysis identified AES and BDI-2 baseline scores as significant predictors of a negative subjective outcome. Cut-off scores of $36 / 37$ for the AES and of 16/17 for the BDI-2 are being suggested.

Considering our results, it has to be questionable whether group-neg patients should be considered as candidates for STN-DBS, even though they fulfilled the formal criteria for DBS surgery, according to leading expert's opinions. ${ }^{19} 33$ Group-neg had the best preoperative motor on-state compared with the 


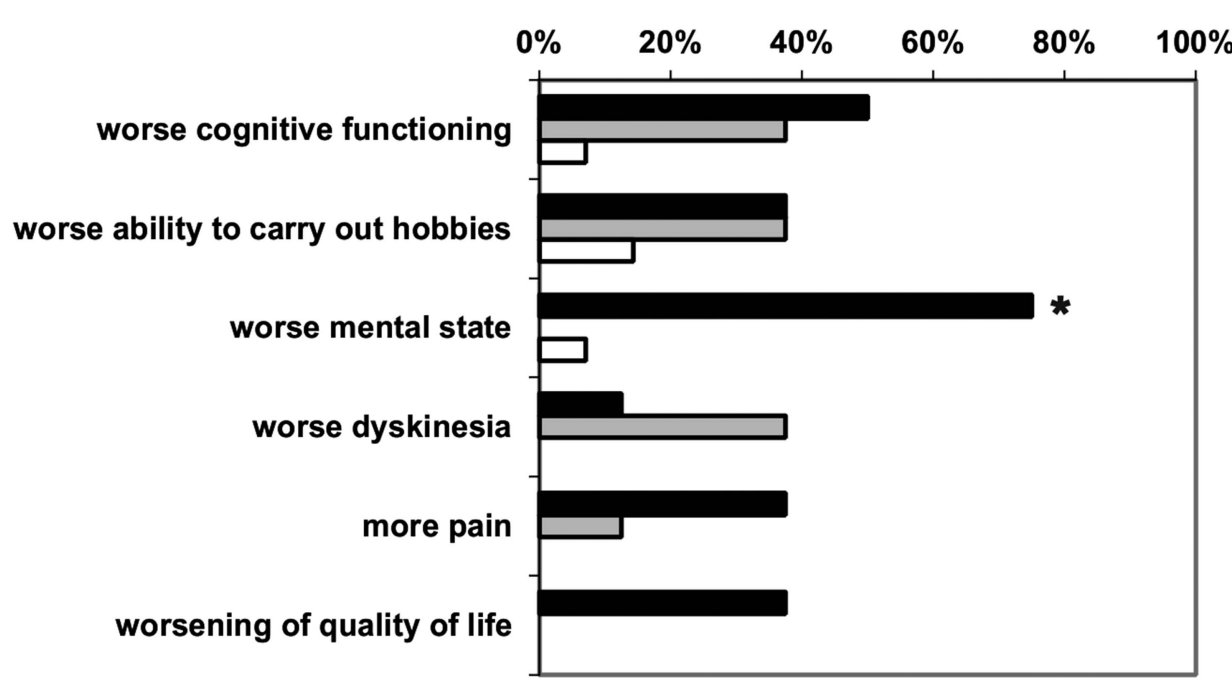

Figure 3 Negative statements at 3 months after STN-DBS for the three groups. All patients were asked to reflect their subjective outcome regarding STN-DBS in a semistructured interview. Negative statements were sorted according to their frequency of being stated by all patients and were then classified concerning the three subjective outcome groups. Included were only such statements which were named by $>25 \%$ of patients within one of the three groups. *Significant difference between the subjective negative outcome group and the other two groups.

other groups. Therefore, it might have been better to continue treatment exclusively with antiparkinsonian medication. However, despite their subjective negative outcome, group-neg experienced significant motor improvements by STN-DBS as measured with the UPDRS-III. Also, their LEDD decreased significantly from baseline to $3 \mathrm{mFU}$. Therefore, these patients cannot be considered as 'motor non-responders'. Still, group-neg did not improve in QoL and even worsened in verbal fluency.

Table 2 Neurological and neuropsychological data, and quality of life for the three groups at baseline and $3 \mathrm{mFU}$

\begin{tabular}{|c|c|c|c|c|c|c|c|}
\hline & & & Group-neg $(n=8)$ & Group-mix $(n=8)$ & Group-pos $(n=14)$ & $\mathrm{F} / \chi^{2}$ & p Value \\
\hline \multicolumn{8}{|l|}{ Neurological data } \\
\hline UPDRS-III & Med on & Baseline & $16.00 \pm 4.14$ & $20.88 \pm 7.36$ & $23.57 \pm 12.75$ & $2.333^{\mathrm{b}}$ & 0.312 \\
\hline \multirow[t]{2}{*}{ UPDRS-III } & Med off & Baseline & $39.88 \pm 7.97$ & $40.00 \pm 11.89$ & $46.79 \pm 16.09$ & $0.985^{a}$ & 0.386 \\
\hline & Stim on and med off & $3 \mathrm{mFU}$ & $28.63 \pm 10.47^{* *}$ & $28.13 \pm 9.13^{*}$ & $24.77 \pm 18.11^{* * *} \S$ & $0.232^{\mathrm{a}}$ & 0.795 \\
\hline \multirow[t]{2}{*}{ LEDD (mg) } & & Baseline & $900.75 \pm 576.63$ & $806.25 \pm 371.63$ & $709.86 \pm 340.60$ & $0.534^{\mathrm{a}}$ & 0.593 \\
\hline & & $3 \mathrm{mFU}$ & $479.58 \pm 299.94^{*}$ & $250.42 \pm 243.01^{* *}$ & $326.36 \pm 116.41$ ** & $2.000^{\mathrm{b}}$ & 0.368 \\
\hline \multicolumn{8}{|l|}{ Neuropsychological data } \\
\hline \multirow[t]{2}{*}{ Digit span forwards T-scores } & & Baseline & $58.13 \pm 9.46$ & $57.86 \pm 9.25 \S$ & $53.86 \pm 11.26$ & $0.581^{\mathrm{a}}$ & 0.566 \\
\hline & & $3 \mathrm{mFU}$ & $52.50 \pm 13.31$ & $53.25 \pm 5.87$ & $49.79 \pm 12.59$ & $0.280^{\mathrm{a}}$ & 0.758 \\
\hline \multirow[t]{2}{*}{ Digit span backward T-scores } & & Baseline & $49.00 \pm 11.59$ & $43.43 \pm 6.13 \S$ & $47.14 \pm 9.35$ & $0.677^{\mathrm{a}}$ & 0.517 \\
\hline & & $3 \mathrm{mFU}$ & $49.13 \pm 12.68$ & $41.88 \pm 19.78$ & $45.15 \pm 7.16$ & $0.866^{\mathrm{b}}$ & 0.649 \\
\hline \multirow[t]{2}{*}{ Verbal fluency (letters) T-scores } & & Baseline & $54.58 \pm 8.75$ & $47.67 \pm 9.06 \S$ & $49.93 \pm 10.25$ & $1.050^{\mathrm{a}}$ & 0.364 \\
\hline & & $3 \mathrm{mFU}$ & $47.87 \pm 6.88^{*}$ & $46.63 \pm 9.95$ & $50.02 \pm 10.95$ & $0.333^{\mathrm{a}}$ & 0.720 \\
\hline \multirow[t]{2}{*}{ Brief test of attention raw scores } & & Baseline & $11.88 \pm 4.12$ & $9.86 \pm 3.53 \S$ & $10.14 \pm 4.69$ & $0.535^{\mathrm{a}}$ & 0.592 \\
\hline & & $3 \mathrm{mFU}$ & $9.00 \pm 4.14$ & $11.25 \pm 3.06$ & $10.07 \pm 4.83$ & $0.560^{\mathrm{a}}$ & 0.578 \\
\hline \multicolumn{8}{|l|}{ Quality of life } \\
\hline \multirow[t]{2}{*}{ PDQ-39 } & & Baseline & $47.09 \pm 19.46$ & $34.71 \pm 16.05$ & $33.58 \pm 13.55$ & $2.001^{a}$ & 0.155 \\
\hline & & $3 \mathrm{mFU}$ & $43.17 \pm 14.01 \dagger, \ddagger$,§ & $20.44 \pm 9.28 t^{*}$ & $21.56 \pm 12.06 \ddagger^{* *}$ & $9.143^{a}$ & 0.001 \\
\hline
\end{tabular}

Values represent means \pm SD. $p$ Values by ${ }^{a}$ ANOVA (F) or ${ }^{b}$ Kruskall-Wallis H-test $\left(\chi^{2}\right)$.

* Represent significant changes for paired samples $t$ test for UPDRS-III (baseline med off vs $3 \mathrm{mFU}$ stim on/med off), LEDD, PDQ-39 and verbal fluency data within a group,

${ }^{*}<0.05,{ }^{* *}<0.01,{ }^{* * *}<0.001$.

†Bonferroni posthoc test: significant difference between group-neg and group-mix.

$\ddagger$ Bonferroni posthoc test: significant difference between group-neg and group-pos.

$\S$ Missing data for one patient.

$\mathrm{N}$, number of patients; group-neg, subjective outcome negative; group-mix, subjective outcome mixed; group-pos, subjective outcome positive; med off, medication off; stim on, stimulation on; 3 mFU, 3 months follow-up; UPDRS, Unified Parkinson's Disease Rating Scale-III; LEDD, I-dopa equivalent daily dose; PDO-39, Parkinson's Disease Questionnaire-39.

Test score range: UPDRS-III, 0-108, higher scores equal more impairment; digit span forwards, $0-100$, higher scores equal better performance; digit span backwards, $0-100$, higher scores equal better performance; verbal fluency (letters), $0-100$, higher scores equal better performance; brief test of attention, $0-20$, higher scores equal better performance; PDQ-39,

$0-100$, higher scores equal more impaired quality of life. 
Table 3 Group comparisons of mood scales at baseline and 3 months after surgery

\begin{tabular}{|c|c|c|c|c|c|}
\hline & Group-neg $(n=8)$ & Group-mix $(n=8)$ & Group-pos $(n=14)$ & $\mathbf{F}$ & p Value \\
\hline \multicolumn{6}{|c|}{ Apathy evaluation scale } \\
\hline Baseline & $45.25 \pm 11.52 t, \ddagger$ & $29.88 \pm 8.59 \dagger$ & $31.64 \pm 7.85 \ddagger$ & 7.251 & 0.003 \\
\hline $3 \mathrm{mFU}$ & $44.50 \pm 7.15 t, \ddagger$ & $27.00 \pm 6.63 \dagger$ & $33.31 \pm 6.85 \ddagger$ & 13.465 & $<0.001$ \\
\hline \multicolumn{6}{|c|}{ Beck depression inventory-2 } \\
\hline Baseline & $18.25 \pm 8.70 \dagger$ & $6.63 \pm 6.28 t$ & $11.43 \pm 4.89$ & 6.626 & 0.005 \\
\hline $3 \mathrm{mFU}$ & $18.38 \pm 5.83 \dagger, \ddagger$ & $5.88 \pm 9.19 \dagger$ & $7.36 \pm 6.86 \ddagger^{*}$ & 7.541 & 0.003 \\
\hline \multicolumn{6}{|c|}{ Self-report manic inventory } \\
\hline Baseline & $7.25 \pm 6.50$ & $2.75 \pm 3.85$ & $7.07 \pm 6.06$ & 1.742 & 0.194 \\
\hline $3 \mathrm{mFU}$ & $11.14 \pm 6.62 \S$ & $3.75 \pm 3.28$ & $10.14 \pm 7.36$ & 3.328 & 0.052 \\
\hline \multicolumn{6}{|c|}{ State trait anxiety inventory state } \\
\hline Baseline & $49.63 \pm 14.44$ & $34.63 \pm 9.40$ & $40.14 \pm 8.99$ & 4.009 & 0.030 \\
\hline $3 \mathrm{mFU}$ & $47.00 \pm 7.64 \S$ & $34.13 \pm 11.84$ & $35.14 \pm 8.87$ & 4.413 & 0.022 \\
\hline \multicolumn{6}{|c|}{$\begin{array}{l}\text { Values represent means } \pm S D \text {. } \\
\text { *Represents significant changes for paired samples } t \text { test for } B D I-2 \text { results within group-pos, }{ }^{*}<0.01 . \\
\text { HBonferroni posthoc test: significant difference between group-neg and group-mix. } \\
\text { ¥Bonferroni posthoc test: significant difference between group-neg and group-pos. } \\
\text { §Missing data for one patient. } \\
\text { Group-neg, subjective outcome negative; group-mix, subjective outcome mixed; group-pos, subjective outcome positive; } 3 \text { mFU, } 3 \text { months follow-up. } \\
\text { Test score range: apathy evaluation scale, 18-72, higher values equal more apathy; Beck depression inventory- } 2,0-63 \text {, higher values equal more depression; self-report manic } \\
\text { inventory, } 0-48 \text {, higher values equal more mania; state trait anxiety inventory, } 20-80 \text {, higher values equal more state anxiety. }\end{array}$} \\
\hline
\end{tabular}

It may seem unexpected, that group-neg presented with best preoperative motor functioning and 1-dopa response, but had the highest apathy and depression scores at the same time. Past studies have shown that apathy and overall motor impairment were not associated, implying an involvement of non-motor frontal-subcortical loops. ${ }^{34}{ }^{35}$ Similar results have been reported for depression and motor impairment, ${ }^{36}$ although this relationship often depends on how depression and its severity was assessed, and what other psychiatric comorbidities existed. ${ }^{37} 38$ Further research is needed to clarify the association between severity of apathy, depression and overall motor symptoms.

As a baseline expectation, group-neg patients significantly more often named 'improvement of mental state', which can usually not be directly improved by DBS, and which might

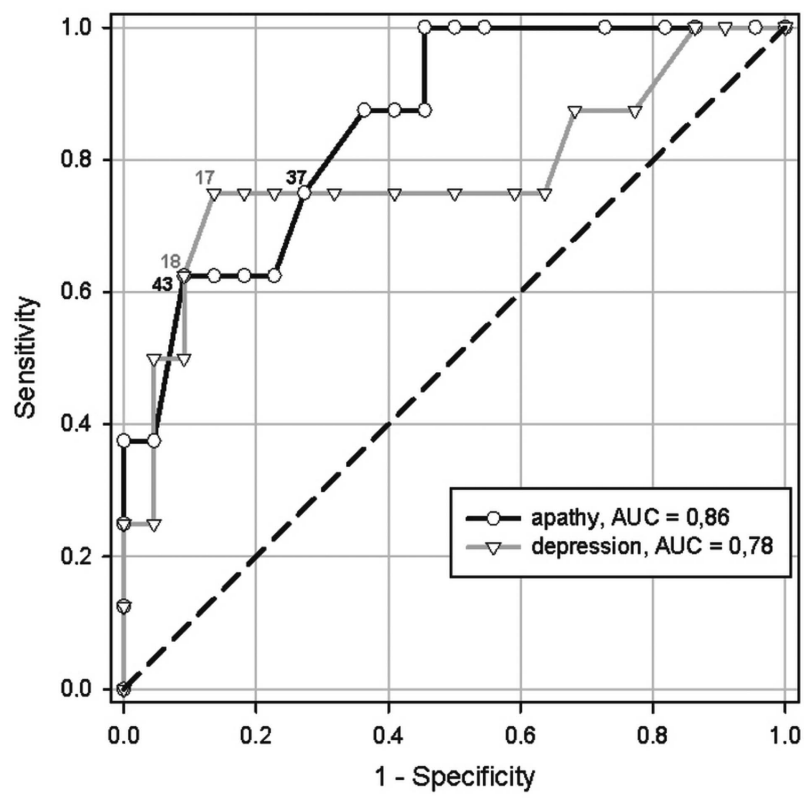

Figure 4 Receiver operating characteristic curves and cut-off scores for apathy (AES) and depression (BDI-2). therefore be considered as unrealistic. Too high and unrealistic expectations can lead to disappointment with DBS outcome, even though patients might have marked motor improvement. ${ }^{39} 40$ Moreover, a patient with more complaints than beneficial changes after DBS is a high burden for the family, the society paying for the procedure, as well as for the involved medical team.

There is an ongoing debate on psychiatric symptoms as possible exclusion criteria for STN-DBS. ${ }^{33}$ Large trials on the efficiency and side effects of STN-DBS usually do not define specific cut-off scores for exclusion but, instead, exclude patients with major or severe psychiatric disorders. ${ }^{1}{ }^{2}$ Also, severe medication-refractory depression is a recommended exclusion criterion for DBS, generally based on DSM-IV diagnosis. ${ }^{41}$ In our study, patients with subjective negative outcome showed significantly more apathy and depression compared with the other groups at baseline and $3 \mathrm{mFU}$, although preoperative severe psychiatric diseases were not found by an experienced psychiatrist. An explanation for the high affective test scores might be l-dopa-induced mood swings or neurovegetative elevation of BDI-2-scores. Nevertheless, apathy and depression are frequent non-motor symptoms in PD. ${ }^{3}$ The relationship between these mood impairments is still unclear. It has been suggested that apathy and depression are discrete components in PD, ${ }^{42}$ while others emphasise an overlap between these two. ${ }^{43}$ Regarding the use of the AES in PD, cut-off scores of 37/38, and for the BDI-2, cut-off scores of $14 / 15$, have been shown to separate patients with apathy/depression from those without. ${ }^{44}{ }^{45}$ With respect to exclusion from DBS therapy, no specific cut-off scores have been defined for the use of these scales. We found cut-off scores of $36 / 37$ on the AES, and of $16 / 17$ on the BDI-2, to be the most sensitive and specific cut-offs, to predict possible subjective negative outcomes in our patient group. This finding suggests that patients should be screened carefully before surgery, and even milder or subclinical psychiatric difficulties should raise our attention with respect to subjective patient outcome and approval to have STN-DBS surgery. Furthermore, apathy and depression should be treated prior to surgery to improve subjective patient outcomes. Also, counselling or psychotherapy accompanying the DBS-procedure might be helpful to stabilise 
patient's affective state and make sure that expectations can be fulfilled. ${ }^{46}$

Apathy and depression also decrease QoL in PD. ${ }^{17}{ }^{18}$ We assume that the preoperative affective state (ie, high apathy and depression scores) of patients with postoperative negative subjective outcome contributes to the unimproved QoL at $3 \mathrm{mFU}$. We speculate that preoperative apathy and depression prevents improvement of QoL after surgery. Regarding neuropsychological results, a worsening in verbal fluency, especially in the more depressed group-neg, is not surprising, since this is a frequent cognitive change after STN-DBS. ${ }^{33}$

Our study has several limitations. The small sample size may limit statistical results and power. However, the very timeconsuming assessment, transcription and evaluation of semistructured interviews usually reduce the number of patients. The use of semistructured interviews makes a comparison between patients more difficult since each person answers questions according to their own perception. Therefore, the objectivity of our interview results is reduced. Moreover, neutral interview responses were not considered in the analysis, which could lead to loss of information. Another limitation is the early follow-up examination at 3 months after surgery. Until now, we were not able to make an assumption regarding the subjective outcome of groups beyond this time point. Further research is needed to examine patients' subjective perception in the long run.

\section{CONCLUSIONS}

PD patients who undergo STN-DBS have specific hopes and wishes regarding a successful outcome of surgery that does not simply depend on motor improvement. Like others, ${ }^{40}{ }^{41}$ we recommend to discuss expectations with the patient before surgery and highlight probable realistic benefits. It seems that a more careful examination of preoperative depression and apathy, as well as a more limited approval for surgery in the case of moderate psychiatric disorders, increases the patient's subjective positive outcome. Preoperative apathy and depression scores might be helpful tools to identify 'risk candidates' for STN-DBS, providing the opportunity of an accompanying psychosocial support and counselling. Future studies which analyse subjective patient outcomes longitudinally are crucial to get a better understanding of the long-term impact of STN-DBS on a patient's life.

Contributors FM and CJL made substantial contributions to: conception and design, acquisition of data, analysis and interpretation of data; drafting the article and revising it critically for important intellectual content; final approval of the version to be published. NH made substantial contributions to: acquisition of data and analysis; revising it critically for important intellectual content; final approval of the version to be published. $C E, M Z$ and EM made substantial contributions to: analysis and interpretation of data; revising it critically for important intellectual content; final approval of the version to be published. EK, JK and CW made substantial contributions to: conception and design and interpretation of data; revising it critically for important intellectual content; final approval of the version to be published. MM made substantial contributions to: acquisition of data and interpretation of data; revising it critically for important intellectual content; final approval of the version to be published. LT made substantial contributions to: conception and design, acquisition of data and analysis; revising it critically for important intellectual content; final approval of the version to be published.

Funding This study was funded by the German Ministry of Education and Research (BMBF), and by the Canadian Institute of Health Research. This study was part of the international and interdisciplinary project 'Ethical, Legal, and Social Aspects of Deep Brain Stimulation (ELSA-DBS)'. (http://geschichte-ethik.uk-koeln.de/ forschungsstelle-ethik/forschung-1/elsa-dbs). FM and $\mathrm{CL}$ were employed through a grant of the German Parkinson Foundation (Deutsche Parkinson Vereinigung) in 2012 and 2013.

Competing interests $\mathrm{F}$ Maier reports no disclosures. C Lewis reports no disclosures. N Horstkoetter reports no disclosures. C Eggers has received speaker's honoraria from Medtronic Inc. E Kalbe has received honoraria from Novartis and is consultant for Novartis. M Maarouf has received speaker's honoraria from Medtronic Inc. J Kuhn has received honoraria from AstraZeneca, Lilly, Lundbeck and Otsuka Pharma for lecturing at conferences and financial support to travel. J Kuhn received financial support for IIT-DBS studies (not the present investigation) from Medtronic GmbH (Meerbusch, Germany). M Zurowski reports no disclosures. E Moro has received honoraria from Medtronic for consulting services and lecturing. She has also received research support from St Jude Medical, CurePSP, CIHR and educational grant support from Medtronic in the past 12 months. C Woopen reports no disclosures. L Timmermann is consultant for Medtronic Inc, Boston Scientific, Bayer Healthcare, UCB Schwarz Pharma, received honoraria in symposia sponsored by TEVA Pharma, Lundbeck Pharma, Bracco, Gianni PR, Medas Pharma, UCB Schwarz Pharma, Desitin Pharma, Boehringer Ingelheim, GlaxoSmithKline, Eumecom, Orion Pharma, Medtronic, Boston Scientific, Cephalon, Abott, GE Medical. The institution of Professor Timmermann, not Prof Timmermann himself, received funding by the German Research Foundation (DFG) via the Clinical Research Group 219, the German Ministry of Education and Research (BMBF), Manfred und Ursula Müller Stiftung, Klüh Stiftung, Hoffnungsbaum e. V., NBIA DISORDERS SOCIETY USA, the medical faculty of the University of Cologne via the 'Köln Fortune program', Medtronic Inc, and the German Parkinson Foundation (Deutsche Parkinson Vereinigung).

Ethics approval Ethics commission of the University Hospital of Cologne.

Provenance and peer review Not commissioned; externally peer reviewed.

\section{REFERENCES}

1 Deuschl G, Schade-Brittinger C, Krack P, et al. A randomized trial of deep-brain stimulation for Parkinson's disease. N Eng/ J Med 2006;355:896-908.

2 Witt K, Daniels C, Reiff J, et al. Neuropsychological and psychiatric changes after deep brain stimulation for Parkinson's disease: a randomised, multicentre study. Lancet Neurol 2008;7:605-14.

3 Weintraub D, Burn DJ. Parkinson's disease: the quintessential neuropsychiatric disorder. Mov Disord 2011;26:1022-31.

4 Jenkinson C, Fitzpatrick R, Peto V, et al. The Parkinson's Disease Questionnaire (PDQ-39): development and validation of a Parkinson's disease summary index score. Age Ageing 1997;26:353-7.

5 Diamond A, Jankovic J. The effect of deep brain stimulation on quality of life in movement disorders. J Neurol Neurosurg Psychiatry 2005;76:1188-93.

6 Schupbach M, Gargiulo M, Welter ML, et al. Neurosurgery in Parkinson disease: a distressed mind in a repaired body? Neurology 2006;66:1811-16.

7 Drapier S, Raoul S, Drapier D, et al. Only physical aspects of quality of life are significantly improved by bilateral subthalamic stimulation in Parkinson's disease. J Neurol 2005;252:583-8.

8 Montel SR, Bungener C. Coping and quality of life of patients with Parkinson disease who have undergone deep brain stimulation of the subthalamic nucleus. Surg Neurol 2009;72:105-10; discussion 10-1.

9 Nisenzon AN, Robinson ME, Bowers D, et al. Measurement of patient-centered outcomes in Parkinson's disease: what do patients really want from their treatment? Parkinsonism Relat Disord 2011:17:89-94.

10 Bell E, Mathieu G, Racine E. Preparing the ethical future of deep brain stimulation. Surg Neurol 2009;72:577-86; discussion 86.

11 Clausen J. Ethical brain stimulation - neuroethics of deep brain stimulation in research and clinical practice. Eur J Neurosci 2010;32:1152-62.

12 Sudmeyer M, Volkmann J, Wojtecki L, et al. Deep brain stimulation —expectations and doubts: a nationwide questionnaire study of patients with Parkinson's disease and their family members. Nervenarzt 2012;83:481-6.

13 Daniels C, Krack P, Volkmann J, et al. Is improvement in the quality of life after subthalamic nucleus stimulation in Parkinson's disease predictable? Mov Disord 2011;26:2516-21.

14 Smeding HM, Speelman JD, Huizenga HM, et al. Predictors of cognitive and psychosocial outcome after STN DBS in Parkinson's Disease. J Neurol Neurosurg Psychiatry 2011;82:754-60.

15 Soulas T, Sultan S, Gurruchaga JM, et al. Depression and coping as predictors of change after deep brain stimulation in Parkinson's disease. World Neurosurg 2011;75:525-32.

16 Witt K, Daniels C, Krack P, et al. Negative impact of borderline global cognitive scores on quality of life after subthalamic nucleus stimulation in Parkinson's disease. J Neurol Sci 2011;310:261-6.

17 Schrag A. Quality of life and depression in Parkinson's disease. J Neurol Sci 2006;248:151-7.

18 Benito-Leon J, Cubo E, Coronell C. Impact of apathy on health-related quality of life in recently diagnosed Parkinson's disease: the ANIMO study. Mov Disord 2012;27:211-18.

19 Hilker R, Benecke R, Deuschl G, et al. Deep brain stimulation for Parkinson's disease. Consensus recommendations of the German Deep Brain Stimulation Association. Nervenarzt 2009:80:646-55. 
20 Kalbe $E$, Kessler J, Calabrese $P$, et al. DemTect: a new, sensitive cognitive screening test to support the diagnosis of mild cognitive impairment and early dementia. Int J Geriatr Psychiatry 2004;19:136-43.

21 Folstein MF, Folstein SE, McHugh PR. 'Mini-mental state'. A practical method for grading the cognitive state of patients for the clinician. J Psychiatr Res 1975; 12:189-98.

22 Mayring P. Qualitative content analysis. Forum: Qualitative Social Research, 2000;1(2).

23 Mayring P. Qualitative Inhaltsanalyse. Grundlagen und Techniken. Weinheim: Beltz, 2008.

24 Fahn S, Elton RL, Committee UD. Unified Parkinson's disease rating scale. In: Fahn S, Marsden CD, Calne D, Goldstein M, eds. Recent developments in Parkinson's disease. Floral Park, NY: MacMillan Healthcare Information, 1987:153-63.

25 Diener HC, Putzki N, eds. Leitlinien für Diagnostik und Therapie in der Neurologie. 4th edn. Stuttgart: Georg Thieme Verlag, 2008.

26 Härting C, Markowitsch HJ, Neufeld H, et al. WMS-R - Wechsler Gedächtnistestrevidierte Fassung. Bern: Huber, 2000.

27 Aschenbrenner S, Tucha O, Lange KW. Regensburger Wortflüssigkeits-Test (RWT). Göttingen: Hogrefe, 2000.

28 Schretlen D, Bobholz JH, Brandt J. Development and psychometric properties of the brief test of attention. Clin Neuropsychologist 1996;10:80-9.

29 Marin RS, Biedrzycki RC, Firinciogullari S. Reliability and validity of the Apathy Evaluation Scale. Psychiatry Res 1991;38:143-62.

30 Beck AT, Steer RA, Ball R, et al. Comparison of Beck Depression Inventories-IA and -II in psychiatric outpatients. J Pers Assess 1996;67:588-97.

31 Shugar G, Schertzer S, Toner BB, et al. Development, use, and factor analysis of a self-report inventory for mania. Compr Psychiatry 1992;33:325-31.

32 Spielberger CD, Gorssuch RL, Lushene PR, et al. Manual for the State-Trait Anxiety Inventory. Consulting Psychologists Press, Inc., 1983.

33 Bronstein JM, Tagliati M, Alterman RL, et al. Deep brain stimulation for Parkinson disease: an expert consensus and review of key issues. Arch Neurol 2011;68:165.
34 Pluck GC, Brown RG. Apathy in Parkinson's disease. J Neurol Neurosurg Psychiatry 2002;73:636-42.

35 Dujardin K, Sockeel P, Devos D, et al. Characteristics of apathy in Parkinson's disease. Mov Disord 2007;22:778-84.

36 Starkstein SE, Preziosi TJ, Bolduc PL, et al. Depression in Parkinson's disease. J Nerv Ment Dis 1990;178:27-31.

37 Starkstein S, Dragovic M, Jorge R, et al. Diagnostic criteria for depression in Parkinson's disease: a study of symptom patterns using latent class analysis. Mov Disord 2011;26:2239-45.

38 Starkstein SE, Brockman S, Hayhow BD. Psychiatric syndromes in Parkinson's disease. Current opinion in psychiatry 2012;25:468-72.

39 Rodriguez RL, Fernandez HH, Haq I, et al. Pearls in patient selection for deep brain stimulation. Neurologist 2007;13:253-60.

40 Okun MS, Rodriguez RL, Foote KD, et al. A case-based review of troubleshooting deep brain stimulator issues in movement and neuropsychiatric disorders. Parkinsonism Relat Disord 2008;14:532-8.

41 Voon V, Saint-Cyr J, Lozano AM, et al. Psychiatric symptoms in patients with Parkinson disease presenting for deep brain stimulation surgery. J Neurosurg 2005; 103:246-51.

42 Kirsch-Darrow L, Marsiske M, Okun MS, et al. Apathy and depression: separate factors in Parkinson's disease. J Int Neuropsychol Soc 2011;17:1058-66.

43 Starkstein SE. Apathy in Parkinson's disease: diagnostic and etiological dilemmas. Mov Disord 2012;27:174-8.

44 Leentjens AF, Dujardin K, Marsh L, et al. Apathy and anhedonia rating scales in Parkinson's disease: critique and recommendations. Mov Disord 2008;23:2004-14.

45 Visser M, Leentjens AF, Marinus J, et al. Reliability and validity of the Beck depression inventory in patients with Parkinson's disease. Mov Disord 2006;21:668-72

46 Woopen C, Timmermann L, Kuhn J. An ethical framework for outcome assessment in psychiatric DBS. AJOB Neuroscience 2012;3:50-5. 